\title{
Simulation of the mechanical behavior of railway ballast by intelligent computing
}

\author{
M. A. Shahin \\ Senior Lecturer, Department of Civil Engineering, Curtin University of Technology, Perth, WA, Australia.
}

\begin{abstract}
Ballast is one of the main components of railway track foundations, thus, an accurate prediction of its mechanical behavior is crucial for stability of railway tracks. In this paper, one of the most commonly used intelligent computing techniques, i.e. artificial neural networks (ANNs), is utilized to model the mechanical behavior of ballast under static loading conditions. Experimental results from a series of large-scale consolidated drained triaxial compression tests collected from the literature are used for ANN model calibration and validation. The results indicate that predictions from the ANN model compare well with those obtained from the large-scale experiments. In particular, ANN predictions demonstrate a high degree of accuracy in simulating the stress-strain and volume change characteristics of ballast. The plastic dilation and contraction of ballast at various confining pressures, and the strain-hardening and post-peak strain-softening are also well simulated.
\end{abstract}

\section{INTRODUCTION}

Ballasted railway tracks are usually consisted of a granular medium of ballast and sub-ballast (capping) placed above a compacted sub-grade (formation soil). The stability and performance of a given railway track are often governed by the mechanical behavior of ballast. Based on experimental results, Indraratna et al. (2001) concluded that ballast can be responsible for more than $60 \%$ of the total deformation of railway tracks, which induces costly regular track maintenance. This necessitates accurate predictions of the constitutive relationships (i.e. stressstrain and volume change under loading) that govern ballast behavior. These constitutive relationships for ballast are very complex and highly nonlinear. Consequently, the development of constitutive models for ballast behavior using conventional analytical solutions requires rigorous mathematical procedures with various model simplifications, which can affect model reliability. An example of such sophisticated mathematical constitutive models for ballast behavior is developed by Salim and Indraratna (2004), which requires 11 ballast parameters that are diffi- cult to determine in the laboratory. In this context, artificial intelligence using neural networks is more efficient as it provides the ballast constitutive model representation, with fewer model parameters, directly from raw experimental laboratory data without any need for problem simplifications or assumptions.

The potential use of neural networks for constitutive modeling was first introduced by Ghaboussi et al. (1991) and since then, neural networks have been applied successfully in constitutive modeling of soils (e.g. Ellis et al., 1995; Penumadu and Zhao, 1999; Zhu et al., 1998). In this study, the feasibility of using artificial neural network in developing accurate and parsimonious constitutive models for ballast behavior is investigated.

\section{BRIEF OVERVIEW OF ARTIFICIAL NEURAL NETWORKS}

The type of artificial neural networks (ANNs) used in this study are multilayer perceptrons (MLPs) that are trained with the back-propagation algorithm 
(Rumelhart et al., 1986). A comprehensive description of back-propagation MLPs is beyond the scope of this paper but can be found in Fausett (1994).

The typical MLP consists of a number of processing elements or nodes that are arranged in layers: an input layer; an output layer; and one or more intermediate layers called hidden layers. Each processing element in a specific layer is linked to the processing element of the other layers via weighted connections. The input from each processing element in the previous layer is multiplied by an adjustable connection weight. The weighted inputs are summed at each processing element, and a threshold value (or bias) is either added or subtracted. The combined input is then passed through a nonlinear transfer function (e.g. sigmoidal or tanh function) to produce the output of the processing element. The output of one processing element provides the input to the processing elements in the next layer. The propagation of information in MLPs starts at the input layer, where the network is presented with a pattern of measured input data and the corresponding measured outputs. The outputs of the network are compared with the measured outputs, and an error is calculated. This error is used with a learning rule to adjust the connection weights to minimize the prediction error. The above procedure is repeated with presentation of new input and output data until some stopping criterion is met. Using the above procedure, the network can obtain a set of weights that produces input-output mapping with the smallest possible error. This process is called "training" or "learning". Once training has been successful, the performance of the trained model has to be verified using an independent validation set.

\section{CONSTITUTIVE MODELLING OF BALLAST USING ARTIFICIAL NEURAL NETWORKS}

In this work, the artificial neural networks for constitutive modeling of ballast are developed using the computer-based software package Neuframe Version 4 (Neusciences, 2000). The data used to calibrate and validate the models consisted of results from six large-scale triaxial, isotropically consolidated compression tests that were reported by Indraratna et al. (1998). The ballast used was latite basalt, a quarried igneous aggregate that is commonly used as railway ballast in New South Wales, Australia. Latite basalt used is highly angular in shape and has coefficient of uniformity $\mathrm{C}_{\mathrm{u}}=1.5$, coefficient of curvature $C_{c}=0.9$, unit weight $\gamma=15.3 \mathrm{kN} / \mathrm{m}^{3}$, maximum particle size $d_{\max }=53 \mathrm{~mm}$ and effective particle size $\mathrm{d}_{10}=27.1 \mathrm{~mm}$. The triaxial compression tests were conducted under drained conditions at confining pressures between 15 to $240 \mathrm{kPa}$.

\subsection{Model inputs and outputs}

In simulations of the mechanical behavior of soils and rocks, e.g. ballast, the current state of stress and strain governs the next state of stress and strain. Thus, a typical neural network for constitutive modeling of ballast includes current state nodes, which are processing element that remember past activity (i.e. memory nodes). At the beginning of the training process, the inputs for current state of stress and/or strain are set to zero and training proceeds to predict the next expected state of stress and/or strain for an input strain or stress increment. The predicted stress and/or strain are then copied back to the current state nodes for the next pattern of input data.

In this work, two single-output ANN models are developed to simulate the stress-strain and volume change characteristics of ballast. The inputs to the first ANN model are the current state of deviator stress $\left(\mathrm{q}_{\mathrm{i}}\right)$, confining pressure $\left(\sigma_{3}\right)$, current axial strain $\left(\varepsilon_{\mathrm{a}, \mathrm{i}}\right)$ and axial strain increment $\left(\Delta \varepsilon_{\mathrm{a}, \mathrm{i}}\right)$. The single output is the next state of stress $\left(\mathrm{q}_{\mathrm{i}+1}\right)$. The inputs of the second ANN model are the current state of volumetric strain $\left(\varepsilon_{\mathrm{v}, \mathrm{i}}\right)$, confining pressure $\left(\sigma_{3}\right)$, current axial strain $\left(\varepsilon_{\mathrm{a}, \mathrm{i}}\right)$ and axial strain increment $\left(\Delta \varepsilon_{\mathrm{a}, \mathrm{i}}\right)$. The single output is the next state of volumetric strain $\left(\varepsilon_{\mathrm{v}, \mathrm{i}+1}\right)$. In this study, the following varying axial strain increments are chosen: $0.05,0.1$, $0.2,0.3, \ldots, 1.1,1.2,1.3, \ldots, 1.8,1.9,2.0$. As recommended by Penumadu and Zhao (1999), using varying strain increment values results in good modeling capability without the need for a large size of training data. Because the data needed for the ANN models at the above strain increments were not recorded in the original experiments of the triaxial tests, the curves of the deviator stress-axial strain and volumetric strain-axial strain of the available triaxial tests were digitized to obtain the required data. A set of 21 training patterns was used in representing a single triaxial test.

\subsection{Data division and scaling}

The six available triaxial tests were divided into two sets: training set for model calibration and an independent validation set for model verification. The training set includes the triaxial tests results related to confining pressures of 15, 60, 120 and 240 $\mathrm{kPa}$; whereas the testing set has two triaxial test results at confining pressures of 30 and $90 \mathrm{kPa}$. Before presenting the data patterns to the neural networks, the inputs and outputs are scaled between 0.0 and 1.0 to eliminate their dimensions and to ensure that they all receive equal attention during training. 


\subsection{Model architecture and internal parameters}

Model architecture requires selection of the optimum number of hidden layers and the corresponding number of hidden nodes. As proposed by Hornik (1989) and Cybenko (1989), a network with one hidden layer can approximate any continuous function if sufficient connection weights are used. Therefore, one hidden layer was used in the current study. The optimal number of hidden nodes is obtained by a trial-and-error approach in which the network was trained using the software default values of learning rate of 0.2 and momentum term of 0.8 , with a tanh transfer function in the hidden layer nodes and a sigmoidal transfer function in the output layer node. For each selected number of hidden layer nodes, training is terminated when the error between the actual and predicted values of outputs over all patterns has no significant improvement. This was achieved at 20,000 training cycles (epochs). As a result of training, a network with three hidden layer nodes was found to perform the best for the deviator stress-axial strain model; whereas a network with two hidden layer nodes was optimal for the volumetric strain-axial strain model.

\subsection{Model performance and validation}

The performance of the developed ANN models in the training set is shown in Figure 1, and the predictive ability of the models in the validation set is depicted in Figure 2. It can be seen that excellent agreement has been achieved between ANN model predictions and laboratory experimental data in the training and validation sets, with coefficients of correlation equal to unity in both sets. This demonstrates the strong capability of ANN models in generalizing the complex nonlinear constitutive relationships of ballast behavior. For example, the nonlinear relationships of deviator stress versus axial strain and volumetric strain (compression is considered positive and dilation is negative) versus axial strain are predicted accurately. The strain hardening and the gradual decrease of deviator stress beyond peak failure (post-peak strain softening) are very well simulated. The transition of ballast behavior from initial compression to dilation at low confining pressures and the change from dilative behavior at low confining pressure to overall compacting behavior at high confining pressure are also well captured. In conventional constitutive modeling, the strain softening region will result in negative soil modulus, which tends to increase the mathematical modeling effort significantly (Zhu et al., 1998).

As mentioned earlier, the current state of stress and strain affects the next state of stress and strain. Consequently, in modeling the ANN constitutive relationships of ballast behavior, an approach was used to add incremental axial strain to the current
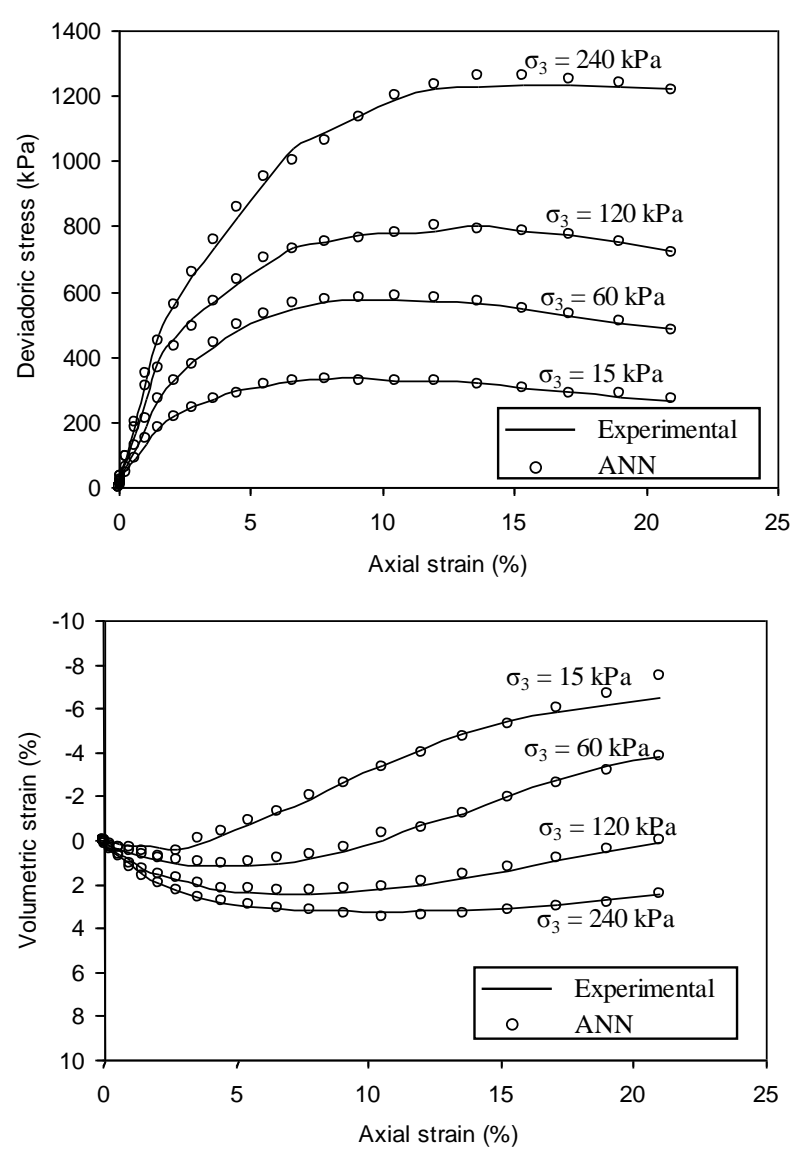

Figure 1. Performance of the developed ANN models in the training set
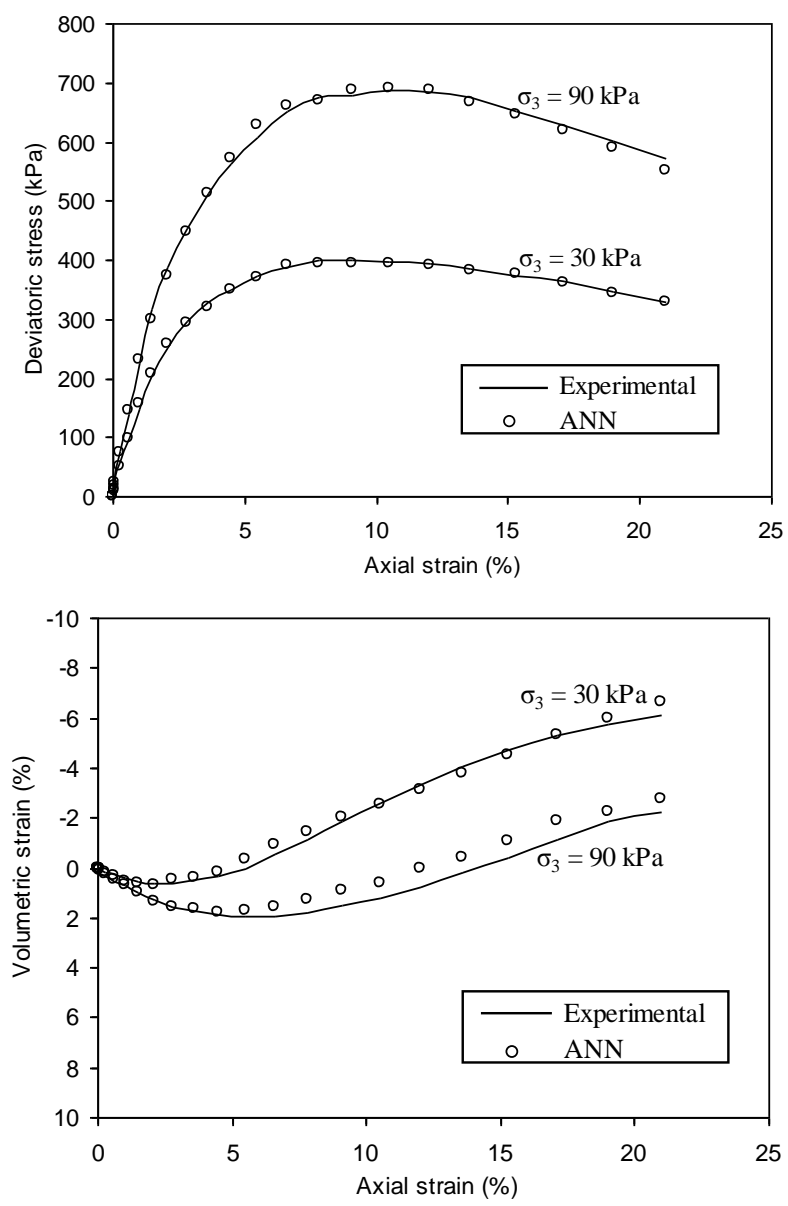

Figure 2. Predictive ability of the developed ANN models in the validation set 
stress and strain so that the next stress and strain are predicted, which are copied back to the current state of stress and strain for the next pattern of input data. The above procedure is applied using the developed ANN models at confining pressures of 30 and 90 $\mathrm{kPa}$, and the virtual results, which are shown in Figure 3 , are compared with the experimental laboratory data. It can be seen from Figure 3 that good agreement still exists between the measured and predicted deviator stress-axial strain, and volumetric strain-axial strain.
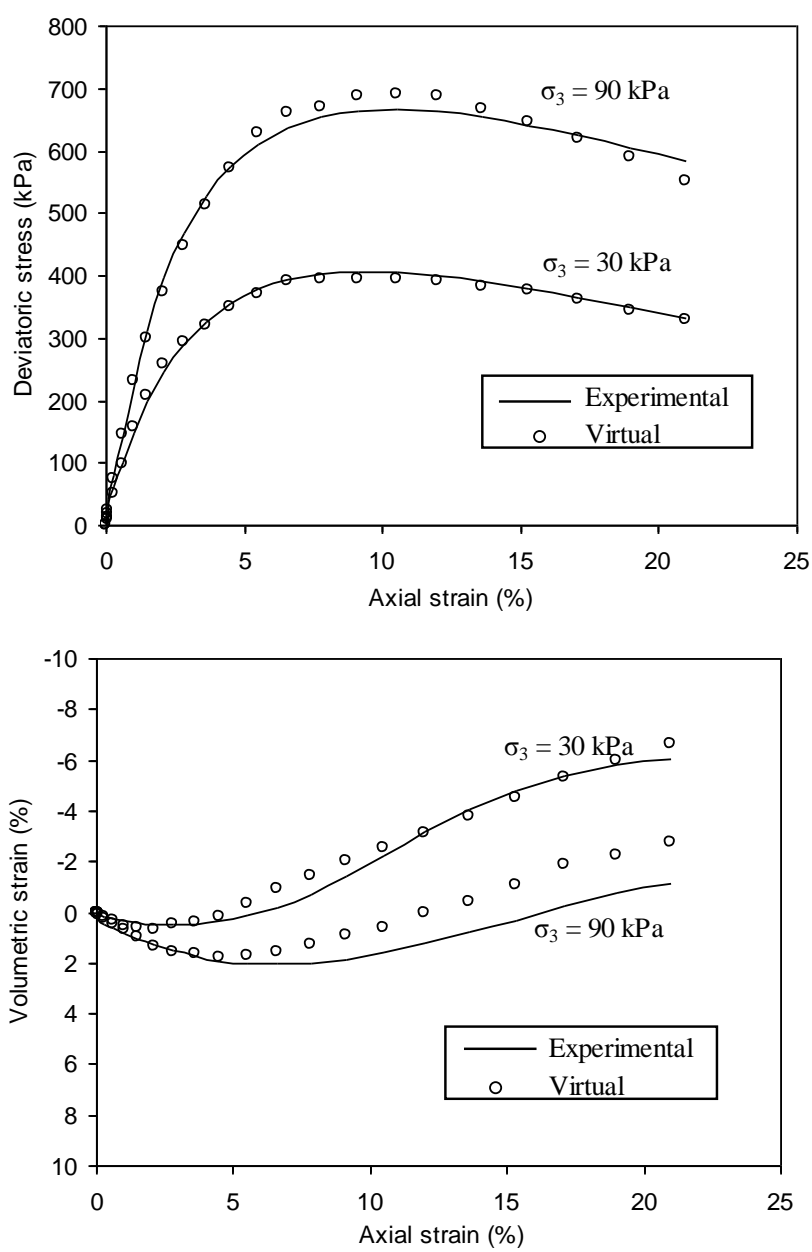

Figure 3. True predictions of the developed ANN models in the validation sets

\section{SUMMARY AND CONCLUSIONS}

Artificial neural networks (ANNs) were used to model the constitutive relationships of the mechanical behavior of railway ballast. Two ANN models were developed; one to simulate the deviator stressaxial strain behavior and the other for volumetric strain-axial strain behavior. The type of ANNs used were multilayer perceptrons (MLPs) that were trained with the back-propagation algorithm. The scheme used for ANN model development was based on the well known plasticity theory that the current state of stress and/or strain influences the next state of stress and/or strain. The results of the
ANN models were compared with the experimental tests data.

The results indicate that the ANN based models were capable of accurately simulating the complex constitutive relationships of the mechanical behavior of railway ballast. The highly nonlinear relationships of deviator stress versus axial strain and of volumetric strain versus axial strain of ballast at various confining pressures were accurately predicted. Strain hardening and post-peak strain softening were well simulated, and the plastic shear dilation and contraction of ballast were also captured. To facilitate the use of the developed ANN models, they are translated into $\mathrm{C}++$ code that can be implemented in a finite element analysis. The source code can be provided by the author upon request.

\section{REFERENCES}

Cybenko, G. 1989. Approximation by superpositions of a sigmoidal function. Mathematics of Control, Signals, and Systems 3:303-314.

Ellis, G.W., Yao, C., Zhao, R. \& Penumadu, D. 1995. Stressstrain modeling of sands using artificial neural networks. Journal of Geotechnical Engineering 121 (5):429-435.

Fausett, L.V. 1994. Fundamentals neural networks: Architecture, algorithms, and applications. Englewood Cliffs, New Jersey: Prentice-Hall.

Ghaboussi, J., Garrett, J.J. \& Wu, X. 1991. Knowledge-based modeling of material behavior with neural networks. Journal of Engineering Mechanics 117 (1):132-153.

Hornik, K., Stinchcombe, M. \& White, H. 1989. Multilayer feedforward networks are universal approximators. Neural Networks 2:359-366.

Indraratna, B., Ionescu, D. \& Christie, D. 1998. Shear behavior of railway ballast based on large-scale triaxial tests. Journal of Geotechnical and Geoenvironmental Engineering 124 (5):439-449.

Indraratna, B., Salim, W., Ionescu, D. \& Christie, D. 2001. Stress-strain and degradation behavior of railway ballast under static and dynamic loading, based on largescale triaxial testing, Proceedings of the 15th International Conference of Soil Mechanics and Geotechnical Engineering, Istanbul: 2093-2096.

Neusciences. 2000. Neuframe Version 4.0. Southampton, Hampshire: Neusciences Corp.

Penumadu, D. \& Zhao, R. 1999. Triaxial compression behavior of sand and gravel using artificial neural networks (ANN). Computers and Geotechnics 24 (3):207-230.

Rumelhart, D.E., Hinton, G.E. \& Williams, R.J. 1986. Learning internal representation by error propagation. In Parallel Distributed Processing, edited by D. E. Rumelhart \& J. L. McClelland. Cambridge: MIT Press.

Salim, W. \& Indraratna, B. 2004. A new elasto-plastic constitutive model for granular aggregates incorporating particle breakage. Canadian Geotechnical Journal 41 (4):657-671.

Zhu, J.H., Zaman, M.M. \& Anderson, S.A. 1998. Modeling of soil behavior with a recurrent neural network. Canadian Geotechnical Journal 35 (5):858-872. 